\title{
Das PURE Desaster: Vorschnelle Schlagzeilen führen zu unnötiger Verunsicherung von Verbrauchern und Patienten
}

\section{A PURE Disaster: Premature Headlines Lead to Needless Uncertainty of Consumers and Patients}

\section{다(1) $(-)$}

\author{
Autoren \\ Margrit Richter ${ }^{1}$, Sarah Egert ${ }^{1}$, Bernhard Watzl${ }^{2}$, Stefan Lorkowski³ ${ }^{3}$ für die Deutsche Gesellschaft für Ernährung e. V.
} (DGE)

Institute

1 Deutsche Gesellschaft für Ernährung e. V.

2 Institut für Physiologie und Biochemie der Ernährung; Max Rubner-Institut, Bundesforschungsinstitut für Ernährung und Lebensmittel

3 Institut für Ernährungswissenschaften, FriedrichSchiller-Universität Jena und Kompetenzcluster für Ernährung und kardiovaskuläre Gesundheit (nutriCARD) Halle-Jena-Leipzig

\section{Schlüsselwörter}

PURE-Studie, Kohlenhydrate, Fett, Nährstoffrelation, Qualität der Ernährung

Keywords

PURE-Study, carbohydrates, fat, macronutrient composition, nutrition quality

\section{Bibliografie}

DOI https://doi.org/10.1055/a-0603-2782

Aktuel Ernahrungsmed 2018; 43: 173-177

(c) Georg Thieme Verlag KG Stuttgart · New York ISSN 0341-0501

Korrespondenzadresse

Dr. Margrit Richter,

Deutsche Gesellschaft für Ernährung e. V.,

Godesberger Allee 18, 53175 Bonn

richter@dge.de

\section{ZUSAMMENFASSUNG}

Die Auswertung der Prospective Urban Rural Epidemioloy (PURE)-Studie von Dehghan et al. sowie verschiedene, in diesem Zusammenhang veröffentlichte Schlagzeilen suggerierten, dass die Ergebnisse der Studie die gängigen Ernährungsempfehlungen widerlegen und die Empfehlungen dahingehend geändert werden müssten, die Kohlenhydratzufuhr zu senken und die Fettzufuhr zu erhöhen. Durch methodische Schwächen, wie der Einschluss von Probanden mit nichtadäquater Energiezufuhr, den Vergleich mit Referenzgruppen mit unzureichender Nährstoffzufuhr, die Nichtbeachtung der Kohlenhydratqualität, der Ballaststoffzufuhr sowie der Substitution einfacher Kohlenhydrate durch Fett bzw. gesättigte Fettsäuren und die fehlende Stratifizierung der Daten nach Einkommen und Region ist die Aussagekraft der Ergebnisse stark eingeschränkt. Zudem ist die Schlussfolgerung falsch, dass die Erkenntnisse aus der PURE-Studie aktuelle Ernährungsempfehlungen der Deutschen Gesellschaft für Ernährung e.V. (DGE) infrage stellen. Mit einer Ernährung, die entsprechend den Richtwerten der DGE einen moderaten Fettanteil von 30 - 35 En\% und einem Kohlenhydratanteil von über 50 En\% aufweist, ist in der PURE-Studie kein erhöhtes Risiko für Mortalität und Morbidität zu erkennen. Wichtiger als eine Diskussion über Nährstoffrelationen ist aus Sicht der DGE eine generell zu hohe Energiezufuhr und eine unzureichende Qualität der Ernährung, d. h. der zu geringe Verzehr von ballaststoffreichen Lebensmitteln und der zu hohe Verzehr von zugesetztem Zucker und raffinierter Stärke.

\section{ABSTRACT}

The assessment of the Prospective Urban Rural Epidemiology (PURE)-study of Dehghan et al. and several in this context published headlines suggested that global dietary guidelines should be reconsidered towards a decreased carbohydrate intake and increased fat intake. Due to methodological weaknesses like inclusion of participants with inadequate energy intake, use of reference groups with inadequate intake of nutrients, non-observance of carbohydrate quality, intake of dietary fibre, and substitution of monoand disaccharides by dietary fat or saturated fatty acids, as well as missing stratification by income and region, the explanatory power of the results is very limited. The conclusion, that the recommendations of the German Nutrition Society (DGE) may be challenged by the results of the PURE-study is limited. In the PURE-study, a nutrition according to the guiding values of the DGE with a moderate percentage of fat (30-35\% of energy) and more than $50 \%$ of 
energy from carbohydrates indicated no increased risk of morbidity and mortality. According to the DGE, overconsumption of energy in general and insufficient quality of the diet, e.g. inadequate consumption of foods high in dietary fibre and overconsumption of added sugars and refined starch, is more essential than the relation of macronutrients.

\section{Einleitung}

Seit einigen Jahren wird eine lebhafte öffentliche Diskussion um 2 wesentliche Aspekte von Ernährungsempfehlungen geführt. Zum einen geht es um die zu empfehlende Relation der energieliefernden Nährstoffe, konkret um die Anteile von Gesamtfett und Gesamtkohlenhydraten an der Energiezufuhr. Zum anderen wird die Frage diskutiert, ob die Zufuhrmenge an gesättigten Fettsäuren Einfluss auf das Mortalitätsrisiko und das kardiovaskuläre Risiko hat. Trotz der Relevanz dieser Aspekte sind bisher nur wenige qualitativ hochwertige Studien vorhanden, die einen Beitrag zur Klärung dieser Sachverhalte leisten.

Am 29. August 2017 veröffentlichte die Zeitschrift The Lancet auf ihrer Website eine Auswertung der Prospective Urban Rural Epidemioloy (PURE)-Studie [1]. Darin wurde die Assoziation der Fett- und Kohlenhydratzufuhr mit der Gesamtmortalität und dem kardiovaskulären Risiko untersucht. Zusätzlich wurde die Assoziation der gesättigten sowie der einfach und mehrfach ungesättigten Fettsäuren mit den zuvor genannten Endpunkten ausgewertet.

\section{Wesentliche Ergebnisse der PURE-Studie und Schlussfolgerungen der Autoren}

In der Analyse der PURE-Studie [1] wurden die Daten von 135335 Probanden aus 18 Ländern ausgewertet. Die Kollektive stammten von allen Kontinenten und repräsentierten Länder mit niedrigem (4), mittlerem (11) und hohem (3) Einkommen. Die Probanden waren im Alter von 35-70 Jahren. Die mittlere Nachbeobachtungszeit betrug 7,4 Jahre. Die Probanden füllten bei Studieneintritt einen Fragebogen zur Verzehrhäufigkeit bestimmter Lebensmittel aus. Als plausibel wurde eine Energiezufuhr von $500-5000 \mathrm{kcal} / \mathrm{d}$ angesehen. Aus den erhobenen Daten wurde die Zufuhr der energieliefernden Nährstoffe in Prozent der Nahrungsenergie (En\%) bestimmt. Anschließend wurden die Probanden für die Nährstoffgruppen Gesamtkohlenhydrate, Gesamtprotein, Gesamtfett, gesättigte sowie einfach und mehrfach ungesättigte Fettsäuren in Quintilen der Zufuhr eingeteilt. Berechnet wurde die Hazard Ratio (HR) aller Quintile im Vergleich zum Referenzquintil für Gesamtmortalität, kardiovaskuläre Mortalität, nicht-kardiovaskuläre Mortalität, schwere kardiovaskuläre Ereignisse, Herzinfarkt sowie Schlaganfall. Als Referenz wurde jeweils das Quintil mit der niedrigsten Zufuhr der betreffenden Nährstoffgruppe verwendet.

Innerhalb der Nachbeobachtungszeit traten 5796 Todesfälle und 4784 schwere kardiovaskuläre Ereignisse auf. Personen mit der höchsten Zufuhr an Kohlenhydraten (77,2 En\%) hatten im Vergleich zu Personen mit der geringsten Kohlenhydratzufuhr $(46,4 \mathrm{En} \%)$ ein höheres Risiko für Gesamtmortalität und nichtkardiovaskuläre Mortalität. Es gab keine Assoziationen zwi- schen Kohlenhydratzufuhr und kardiovaskulären Ereignissen sowie kardiovaskulärer Mortalität.

Personen mit der geringsten Fettzufuhr (10,6 En\%) hatten im Vergleich zu Personen mit der höchsten Fettzufuhr (35,3 En\%) ein geringeres Risiko für Gesamtmortalität sowie für nicht-kardiovaskuläre Mortalität. Es gab keine Assoziationen zwischen Fettzufuhr und kardiovaskulären Ereignissen oder kardiovaskulärer Mortalität. Die Assoziationen zwischen der Zufuhr von Protein sowie gesättigten, einfach und mehrfach ungesättigten Fettsäuren und den gewählten Endpunkten waren ähnlich wie die zwischen Fettzufuhr und den untersuchten Endpunkten.

Die Autoren schließen daraus, dass ihre Ergebnisse Ernährungsempfehlungen, die eine Reduktion des Anteils von Gesamtfett auf unter $30 \mathrm{En \%}$ und gesättigten Fettsäuren auf unter 10 En\% empfehlen, nicht unterstützen. Auch sei es unwahrscheinlich, dass eine Beschränkung des allgemeinen Fettkonsums die Gesundheit der Bevölkerung verbessert. Die Autoren folgern ferner, dass Personen mit einer hohen Kohlenhydratzufuhr von mehr als 60 En\% von einer Reduktion der Kohlenhydratzufuhr und einer Erhöhung der Fettzufuhr profitieren würden. Am besten eigne sich eine ausgewogene Kost, die zu etwa 50 - 55 En\% aus Kohlenhydraten und zu rund 35 En\% aus Fett bestehe; die 35 En\%-Fettzufuhr umfassen sowohl ungesättigte als auch gesättigte Fettsäuren [1]. Mit diesen Schlussfolgerungen bestätigen die Autoren die Aussagen der Deutschen Gesellschaft für Ernährung e. V. (DGE) zur Nährstoffrelation. Die DGE spricht sich für einen moderaten Fettanteil von 30-35 En\% und einen Kohlenhydratanteil von über 50 En\% aus [2, 3].

\section{Kommunikation der PURE-Ergebnisse in den Medien}

Die Auswertung der PURE-Studie [1] hat weltweit für viel mediale Aufmerksamkeit gesorgt. Im Anschluss an die Veröffentlichung der Studienergebnisse erschienen zahlreiche Artikel dazu in der Fachpresse (z. B. Medscape [4], ÄrzteZeitung online [5]). Auch die Laienpresse griff die Studie auf (z. B. Bild [6], Focus online [7]). Die Schlagzeilen suggerierten, dass die Ergebnisse der PURE-Studie die gängigen Ernährungsempfehlungen widerlegen und die Empfehlungen dahingehend geändert werden müssten, die Kohlenhydratzufuhr zu senken und die Fettzufuhr zu erhöhen. Doch wie ist dies wissenschaftlich zu beurteilen? Widerlegen die Studienergebnisse tatsächlich gängige Ernährungsempfehlungen wie bspw. die der DGE? Kann eine einzelne Kohortenstudie die evidenzbasierten Aussagen von wissenschaftlichen Leitlinien überhaupt komplett widerlegen? Um dies zu beurteilen, müssen die Studie, die eingesetzte Methodik und die Ergebnisse sowie die daraus gezogenen Schlussfolgerungen kritisch betrachtet werden. 


\section{Methodische Schwächen der PURE-Studie}

Das PURE-Konsortium hat eine beachtliche Datenbasis erhoben, welche vor allem die Ernährungssituation und die gesundheitlichen Probleme in Ländern mit mittlerem und geringem Einkommen abbildet. Allerdings weisen sowohl die Datenauswertung als auch die Interpretation gravierende Mängel auf (siehe z.B. [8]), die zu falschen Schlussfolgerungen geführt haben. Die Autoren weisen selbst darauf hin, dass mit der nur einmaligen Erhebung der Ernährung keine ausreichenden Daten vorliegen [1]. Aufgrund der einmaligen Datenerhebung bleibt unklar, ob sich die Ernährung während der Nachbeobachtungszeit verändert hat.

\section{Einschluss von Probanden mit nicht adäquater Energiezufuhr}

Die Autoren stuften eine Energiezufuhr von $500-5000 \mathrm{kcal} / \mathrm{d}$ als plausibel ein [1]. Der D-A-CH-Referenzwert für die durchschnittliche tägliche Energiezufuhr für Erwachsene liegt bei geringer körperlicher Aktivität bei $1700-2400$ kcal [9]. Eine Energiezufuhr von $500 \mathrm{kcal} / \mathrm{d}$ ist bei Erwachsenen unzureichend und resultiert sowohl in einer quantitativen (Unterernährung) als auch qualitativen (Fehlernährung) Mangelernährung. Neben einer nichtadäquaten Energiezufuhr, ist auch eine unzureichende Versorgung mit essenziellen Nährstoffen wahrscheinlich. Eine Energiezufuhr weit über dem Bedarf verursacht langfristig Übergewicht und erhöht das Risiko für Diabetes mellitus Typ 2 sowie kardiovaskuläre Erkrankungen. Morbidität und Mortalität entstehen in diesen Fällen folglich nicht in erster Linie aufgrund der Verteilung der energieliefernden Nährstoffe, sondern aufgrund einer zu niedrigen oder zu hohen Energiezufuhr sowie ggf. einer unzureichenden Versorgung mit essenziellen Nährstoffen. Aus Sicht der DGE ist daher eine Neuauswertung der PURE-Studie mit einem plausibleren Bereich der Energiezufuhr notwendig.

\section{Referenzgruppe mit unzureichender Nährstoff- zufuhr}

In den Auswertungen wird jeweils das erste Quintil der Nährstoffzufuhr als Referenzgruppe für die Risikobestimmung verwendet [1]. Die Fettzufuhr in diesem Quintil ist mit 10,6 En\% sehr niedrig und deutet auf eine extreme und einseitige Ernährungsweise hin. Bei einer derart niedrigen Fettzufuhr ist eine adäquate Zufuhr von essenziellen Fettsäuren sowie von fettlöslichen Vitaminen unwahrscheinlich. Ferner ist in Populationen mit einer hohen Zufuhr an stärkehaltigen Lebensmitteln (Reis, Mais usw.) aufgrund einer an Gemüse, Obst und tierischen Lebensmitteln armen Ernährung meist auch die Zufuhr weiterer Vitamine und Mineralstoffe unzureichend. Das verringerte Krankheits- und Sterblichkeitsrisiko mit steigender Fettzufuhr und einer dadurch insgesamt vollständigeren Nährstoffversorgung kann somit auch auf ein geringeres Risiko für eine qualitative Mangelernährung zurückzuführen sein. Bei der Verwendung des zweiten oder dritten Quintils als Referenzgruppe wären vermutlich keine signifikanten Unterschiede in der Assoziation der Fettzufuhr mit Mortalität und kardiovaskulären Ereignissen innerhalb des Bereiches von 20 - 35 En\% festzustellen.

\section{Nichtberücksichtigung der Kohlenhydratqualität sowie der Substitution einfacher Kohlenhydrate durch Fett bzw. gesättigte Fettsäuren}

Die Qualität der Kohlenhydrate bzw. die Zufuhr von Ballaststoffen wurde in der PURE-Studie [1] nicht berücksichtigt. Eine hohe Kohlenhydratzufuhr ist nicht per se mit einer erhöhten Prävalenz kardiovaskulärer Krankheiten assoziiert. Im Gegensatz zu den Ergebnissen der PURE-Studie zeigt eine weitere Lancet-Publikation bei einem Naturvolk in Bolivien (Tsimane) mit einer Ernährung mit 72 En\% Kohlenhydraten, 14En\% Fett und 14En\% Protein die niedrigste jemals beobachtete Prävalenz von koronaren Herzkrankheiten [10].

Allein die Anteile der energieliefernden Nährstoffe an der zugeführten Energie reichen nicht aus, um die Qualität der Ernährung zu beurteilen. Den Daten im Supplement [11] der PUREPublikation ist zu entnehmen, dass die Kohlenhydrate in den in der Studie untersuchten Kollektiven mit einer besonders hohen Kohlenhydratzufuhr vornehmlich aus stärkehaltigen, raffinierten Grundnahrungsmitteln (Reis, Mais, Weizen) stammten. Eine Ernährung, die zu über 70 En\% aus Kohlenhydraten aus diesen Lebensmitteln besteht, kann den Bedarf an Vitaminen und Mineralstoffen kaum decken [8]. Dies führt wiederum zu einer unzureichenden Versorgung, welche die Morbidität und Mortalität beeinflusst. Frühere Studien haben gezeigt, dass sich eine Verminderung der Zufuhr von raffinierter Stärke und einfachen Zuckern durch eine erhöhte Zufuhr von insbesondere ungesättigten Fettsäuren gesundheitsförderlich auswirkt [12, 13]. Aktuelle Studien belegen zudem, dass eine Ernährung, die reich an Vollkornprodukten bzw. Ballaststoffen ist, das Risiko für z. B. die koronare Herzkrankheit senkt [14], während der Austausch durch gesättigte Fettsäuren ohne Effekt ist $[12,13]$.

In der PURE-Studie ist eine Zufuhr von gesättigten Fettsäuren bis zu 13 En\% nicht mit negativen Auswirkungen auf die untersuchten Endpunkte assoziiert. Daraus schließen die Autoren, dass die Zufuhr von gesättigten Fettsäuren nicht auf < 10 En\% beschränkt werden sollte [1]. Die Ernährungssituation in Ländern mit mittleren und niedrigen Einkommen unterscheidet sich von der in Ländern mit einem hohen Einkommen bezüglich des Verzehrs tierischer Lebensmittel. Bei der Hälfte der 18 beteiligten Länder sind Milchprodukte die primäre Quelle für gesättigte Fettsäuren. Sie sichern aber auch die Versorgung mit kritischen Nährstoffen wie z. B. Protein, B-Vitaminen, Zink und Jod. Eine generelle Aussage zum Risikopotenzial gesättigter Fettsäuren lässt sich aus dieser Auswertung nicht ableiten.

\section{Fehlende Stratifizierung nach Einkommen oder Region}

Verschiedene Studien haben gezeigt, dass die kardiovaskuläre Sterblichkeit in Industrienationen in Abhängigkeit vom ProKopf-Einkommen oder soziodemografischen Index abnimmt, obwohl Risikofaktoren wie z.B. Dyslipidämien, Diabetes mellitus Typ 2 und Adipositas häufiger anzutreffen sind und die Prävalenz kardiovaskulärer Erkrankungen höher ist [15 - 19]. Gründe hierfür sind vermutlich effizientere Gesundheits- und Versorgungssysteme sowie eine verbesserte Prävention und Thera- 
pie [20, 21]. Eine frühere Auswertung der PURE-Daten bestätigt diesen Zusammenhang ebenfalls [22].

Obwohl dieser Zusammenhang bekannt und für die eigenen Daten belegt war, wurden die Daten nicht nach Region oder nach Einkommen stratifiziert ausgewertet. Die gemeinsame Auswertung aller Daten kann Effekte abschwächen. Die Autoren der PURE-Studie haben in weiteren Berechnungen die Assoziation zwischen dem Anteil an Kohlenhydraten und gesättigten Fettsäuren in der Ernährung und der Gesamtsterblichkeit um das Haushaltseinkommen adjustiert; die Ergebnisse unterschieden sich nicht wesentlich von den nicht adjustieren Ergebnissen $[1,11]$. Der Zusammenhang zwischen der prozentualen Nährstoffzufuhr und kardiovaskulären Ereignissen oder kardiovaskulärer Mortalität wurde jedoch nicht adjustiert ausgewertet.

\section{Diskussion}

Von den 135335 Probanden der PURE-Studie stammen lediglich 17000 aus Ländern mit hohem Einkommen (Kanada, Schweden und die Vereinigten Arabischen Emirate) [1]. Die in der Studie beobachteten Effekte sind daher vor allem auf die Länder mit mittlerem und geringem Einkommen zurückzuführen. Ernährung, körperliche Aktivität und Gesundheitsversorgung sind in vielen dieser Länder nicht mit Deutschland vergleichbar. Daher können die Ergebnisse nicht ohne weiteres auf Deutschland übertragen werden.

Die Autoren diskutieren, dass eine erhöhte Fettzufuhr mit einer verminderten Mortalität assoziiert ist und daher eine Beschränkung der Fettzufuhr auf unter 30 En\% aufgehoben werden sollte. Nur wenige in die Auswertung eingeschlossene Kohorten wiesen jedoch eine Fettzufuhr von mehr als 30 En\% auf. Die mittlere Fettzufuhr lag in den einzelnen untersuchten Regionen zwischen 17,7 En\% und 30,5 En\%. Die Probanden im vierten und fünften Quintil der Untersuchung hatten eine Fettzufuhr von 29,1 En\% und 35,3 En\%. Auch das Fettsäurenmuster zeigt, dass die Zufuhr der Probanden im vierten und fünften Quintil den Empfehlungen der DGE entspricht oder geringfügig darüber liegt (gesättigte Fettsäuren: Quintil [Q]4 9,5 En\%, Q5 13,2 En\%; einfach ungesättigte Fettsäuren: Q4 9,5 En\%, Q5 12,5 En\%; mehrfach ungesättigte Fettsäuren Q4 5,7 En\%, Q5 $8,5 \mathrm{En} \%)$. Eine Aussage zu Kostformen mit deutlich mehr als 30 En\% Fett kann aus den Daten nicht verlässlich abgeleitet werden. Im ersten Quintil ist die mediane Fettzufuhr von 10,6 En\% sehr gering. Bei einer so geringen Zufuhr ist eine ausreichende Zufuhr an essenziellen Fettsäuren und fettlöslichen Vitaminen fraglich.

Wichtig ist auch eine differenziertere Betrachtung der Kohlenhydrate. Während raffinierte Kohlenhydrate (v.a. Stärke und zugesetzter Zucker) die Entstehung von Diabetes mellitus Typ 2 und kardiovaskulären Erkrankungen fördern [23-25], ist die Aufnahme komplexer Kohlenhydrate (Ballaststoffe) über wenig verarbeitete Lebensmittel als gesundheitsförderlich anzusehen. Eine ballaststoffreiche Ernährung bzw. eine Ernährung, die reich an Vollkornprodukten ist, kann z. B. das Risiko für koronare Herzkrankheiten [14] und Darmkrebs [26-28] senken. Die Qualität der Kohlenhydrate bzw. die Zufuhr von
Ballaststoffen wurde allerdings von Dehghan et al. [1] nicht untersucht.

Ein großer Teil der Morbidität und Mortalität in Industrieländern wie Deutschland ist einer quantitativen und qualitativen Fehlernährung zuzuschreiben [29]. Es ist jedoch anzuzweifeln, dass eine veränderte Fettzufuhr und eine damit einhergehende Änderung der Nährstoffrelation diese Probleme lösen würden. Nach den Daten der Nationalen Verzehrsstudie II werden in Deutschland bereits im Mittel mindestens 35En\% Fett zugeführt, während die Kohlenhydratzufuhr im Mittel lediglich etwa $45 \mathrm{En} \%$ beträgt [30]; hierbei stellen die Mono- und Disaccharide etwa die Hälfte der zugeführten Kohlenhydrate.

Die PURE-Ergebnisse stellen somit die Aussagen der DGELeitlinien und der DGE-Empfehlungen nicht infrage. Die höhere Mortalität, die in der PURE-Studie mit einer höheren Zufuhr an Kohlenhydraten und einer geringeren Zufuhr an Fett assoziiert ist, geht vermutlich mit einer qualitativ schlechteren Ernährung einher und ist vor allem durch Atemwegserkrankungen und Infektionen bedingt, nicht durch kardiovaskuläre Mortalität [1]. Außerdem stellen die Ergebnisse einer einzelnen prospektiven Kohortenstudie nicht die Ergebnisse einer evidenzbasierten Literaturbewertung infrage. Vielmehr müssen neue Studien bei der turnusgemäßen Überarbeitung von Leitlinien berücksichtigt und vor allem auch hinsichtlich ihrer methodischen Qualität bewertet werden.

\section{Fazit}

Die Auswertung der PURE-Daten [1] weist aus Expertensicht (zum Teil gravierende) methodische Mängel auf. Dadurch ist die Aussagekraft der Ergebnisse stark eingeschränkt. Zudem ist die Schlussfolgerung falsch, dass die Erkenntnisse aus der PURE-Studie aktuelle Ernährungsempfehlungen der DGE infrage stellen oder widerlegen. Mit einer Ernährung, die gemäß den Richtwerten der DGE einen moderaten Fettanteil von 30 35 En\% und einem Kohlenhydratanteil von über 50 En\% aufweist [2, 3], ist in der PURE-Studie kein erhöhtes Risiko für Mortalität und Morbidität zu erkennen.

Abschließend lässt sich aus Sicht der DGE konstatieren, dass Richtwerte für die Energiezufuhr aus Kohlenhydraten und Fett Orientierungswerte darstellen und ein Unterschreiten des Richtwerts für Kohlenhydrate vertretbar ist, wenn entsprechende Voraussetzungen erfüllt sind (für Details siehe [3]). Wichtiger als eine Diskussion über Nährstoffrelationen ist aus Sicht der DGE eine generell zu hohe Energiezufuhr und die Qualität der Ernährung, d.h. der zu geringe Verzehr von ballaststoffreichen Lebensmitteln (bspw. Vollkorn, Hülsenfrüchte, Gemüse, Obst) und der zu hohe Verzehr von einfachen Kohlenhydraten (Mono- und Disaccharide) in Form von zugesetztem Zucker und raffinierter Stärke. 
Interessenkonflikt

Sarah Egert und Margrit Richter sind Mitarbeiterinnen der Deutschen Gesellschaft für Ernährung e.V. (DGE) und in dieser Funktion an der Erstellung verschiedener DGE-Publikationen beteiligt. Bernhard Watzl und Stefan Lorkowski sind Mitglieder des Wissenschaftlichen Präsidiums der DGE. Stefan Lorkowski ist außerdem Mitautor der evidenzbasierten Leitlinie „Fettzufuhr und Prävention ausgewählter ernährungsmitbedingter Krankheiten" (2015) der DGE, war innerhalb der vergangenen 3 Jahre in einem Beratungsgremium der Unternehmen Amgen und Sanofi-Aventis tätig und erhielt Vortragshonorare von AMGEN, Berlin-Chemie, Bund Niedergelassener Kardiologen, Deutsche Veterinärmedizinische Gesellschaft, DGE, Gesellschaft für Prävention \& Rehabilitation HKE Hessen, Klinikum Oldenburg, LÄKT, LAKT, LZKTH, MSD, OmniMed, Sanofi-Aventis, Synlab und Unilever.

\section{Literatur}

[1] Dehghan M, Mente A, Zhang X et al. Associations of fats and carbohydrate intake with cardiovascular disease and mortality in 18 countries from five continents (PURE): a prospective cohort study. Lancet 2017; 390: 2050-2062

[2] Deutsche Gesellschaft für Ernährung, Österreichische Gesellschaft für Ernährung, Schweizerische Gesellschaft für Ernährung, Hrsg. Referenzwerte für die Nährstoffzufuhr. 2. Aufl., 3. aktualisierte Ausgabe. Bonn: 2017

[3] Deutsche Gesellschaft für Ernährung e. V. (DGE). DGE Position. Richtwerte für die Energiezufuhr aus Kohlenhydraten und Fett. 2011: Im Internet: https://www.dge.de/fileadmin/public/doc/ws/position/ DGE-Positionspapier-Richtwerte-Energiezufuhr-KH-und-Fett.pdf (Stand: 27.03.2018)

[4] Rommelfanger J. PURE mischt gängige Ernährungs-Empfehlungen auf: Fett nicht schädlich, höhere Sterblichkeit unter Kohlenhydraten. (31.08.2017). Im Internet: https://deutsch.medscape.com/artikel ansicht/4906315 (Stand: 28.02.2018)

[5] ÄrzteZeitung online. Fettreiche Kost. Megastudie stellt Empfehlungen zur Ernährung auf den Kopf. (04.09.2017). Im Internet: https:// www.aerztezeitung.de/medizin/krankheiten/herzkreislauf/article/ 942196/fettreiche-kost-megastudie-stellt-empfehlungen-ernaeh rung-kopf.html (Stand: 27.03.2018)

[6] Bild. Fette sind gesünder als Kohlenhydrate! (30.08.2017). Im Internet: http://www.bild.de/ratgeber/gesundheit/diaet/besser-fettessen-53042152.bild.html (Stand: 27.03.2018)

[7] Focus online. Ernährungsempfehlungen wanken. Studie zeigt: So gefährlich sind Kohlenhydrate für die Gesundheit. (07.09.2017). Im Internet: http://www.focus.de/gesundheit/videos/studie-zeigt-soungesund-sind-kohlenhydrate-wirklich_id_7565703.html (Stand: 27.03.2018)

[8] Society of Nutrition and Food Science e.V., Universität Hohenheim. Verlängern mehr Fett und weniger Kohlenhydrate das Leben? - Die fragwürdigen Schlussfolgerungen einer neuen globalen Studie 2017: Im Internet: https://www.uni-hohenheim.de/fileadmin/user_upload/ SNFS_Kommentar_PURE_Studie.pdf (Stand: 27.03.2018)

[9] German Nutrition Society. New reference values for energy intake. Ann Nutr Metab 2015; 66: 219-223

[10] Kaplan H, Thompson RC, Trumble BC et al. Coronary atherosclerosis in indigenous South American Tsimane: a cross-sectional cohort study. Lancet 2017; 389: 1730-1739

[11] Dehghan M, Mente A, Zhang X et al. Supplementary appendix: Associations of fats and carbohydrate intake with cardiovascular disease and mortality in 18 countries from five continents (PURE): a prospective cohort study. Lancet Supplementary appendix; (August
2017). Im Internet: http://www.thelancet.com/cms/attachment/ 2113864408/2084408033/mmc1.pdf (Stand: 27.03.2018)

[12] Wolfram G, Bechthold A, Boeing $H$ et al. Evidence-based guideline of the German Nutrition Society: fat intake and prevention of selected nutrition-related diseases. Ann Nutr Metab 2015; 67: 141 - 204

[13] Lebwohl B, Cao Y, Zong $G$ et al. Long term gluten consumption in adults without celiac disease and risk of coronary heart disease: prospective cohort study. BMJ 2017; 357: j1892

[14] Hauner H, Bechthold A, Boeing $\mathrm{H}$ et al. Evidence-based guideline of the German Nutrition Society: carbohydrate intake and prevention of nutrition-related diseases. Ann Nutr Metab 2012; 60 (Suppl. 01): 1 58

[15] Weir HK, Anderson RN, Coleman King SM et al. Heart disease and cancer deaths - trends and projections in the United States, 19692020. Prev Chronic Dis 2016; 13: E157

[16] Danaei G, Singh GM, Paciorek C] et al. The global cardiovascular risk transition: associations of four metabolic risk factors with national income, urbanization, and Western diet in 1980 and 2008. Circulation 2013; 127: $1493-1502$

[17] Deutsche Herzstiftung, Hrsg. Deutscher Herzbericht 2017. Frankfurt/ M: 2018

[18] Barquera S, Pedroza-Tobías A, Medina C et al. Global overview of the epidemiology of atherosclerotic cardiovascular disease. Arch Med Res 2015; 46: $328-338$

[19] Roth GA, Johnson C, Abajobir A et al. Global, regional, and national burden of cardiovascular diseases for 10 causes, 1990 to 2015. J Am Coll Cardiol 2017; 70: 1-25

[20] Timmis A, Townsend N, Gale C et al. European Society of Cardiology. Cardiovascular disease statistics 2017. Eur Heart ] 2017; 0: 1 - 72

[21] Ford ES, Ajani UA, Croft JB et al. Explaining the decrease in U.S. deaths from coronary disease, 1980 - 2000. N Engl J Med 2007; 356: 2388 2398

[22] Yusuf S, Rangarajan S, Teo K et al. Cardiovascular risk and events in 17 low-, middle-, and high-income countries. N Engl J Med 2014; 371 : $818-827$

[23] Huang C, Huang J, Tian Y et al. Sugar sweetened beverages consumption and risk of coronary heart disease. Atherosclerosis 2014; 234: $11-16$

[24] Xi B, Huang Y, Reilly KH et al. Sugar-sweetened beverages and risk of hypertension and CVD: a dose-response meta-analysis. Br J Nutr 2015; 113: 709-717

[25] Fung TT, Malik V, Rexrode KM et al. Sweetened beverage consumption and risk of coronary heart disease in women. Am J Clin Nutr 2009; 89: $1037-1042$

[26] Haas P, Machado M], Anton AA et al. Effectiveness of whole grain consumption in the prevention of colorectal cancer. Int J Food Sci Nutr 2009; 60 (Suppl. 06): 1- 13

[27] Schatzkin A, Mouw T, Park Y et al. Dietary fiber and whole-grain consumption in relation to colorectal cancer in the NIH-AARP Diet and Health Study. Am J Clin Nutr 2007; 85: $1353-1360$

[28] Aune D, Chan DSM, Lau R et al. Dietary fibre, whole grains, and risk of colorectal cancer: systematic review and dose-response meta-analysis of prospective studies. BMJ 2011; 343: d6617

[29] Statistisches Bundesamt. Gesundheit. Todesursachen in Deutschland 2015. 2017: Im Internet: https://www.destatis.de/DE/Publikationen/ Thematisch/Gesundheit/Todesursachen/Todesursachen2120400 157004.pdf;jsessionid=CEB50F84EDC35EE6945FAA103052B027. InternetLive1?__blob=publicationFile (Stand 27.03.2018)

[30] Krems C, Walter C, Heuer T et al. Lebensmittelverzehr und Nährstoffzufuhr - Ergebnisse der Nationalen Verzehrsstudie II. In: Deutsche Gesellschaft für Ernährung, Hrsg. 12. Ernährungsbericht 2012. Bonn: 2012: $40-85$ 